\title{
Exploración de los componentes de la estructura de la personalidad en desarrollo de niños preescolares con antecedente de prematuridad extrema Exploring the components of the structure of personality in early childhood development with a history of extreme prematurity
}

Virginia Varela-Moraga ${ }^{1,2}$ y Pamela Morales-Dastres ${ }^{3}$

\begin{abstract}
Introduction: Extremely premature children represent a biologically vulnerable population which results from some conditions present at birth: early and prolonged hospitalization in a life-threatening condition. These children often present not only cognitive deficit, but also socialaffective difficulties and impaired infant-parent attachment. Therefore, it could be predicted that there would be adverse consequences in the development of their personality structure. Considering this background, the objective of this study was to identify the characteristics inherent to the components of the structure of personality development in children with a history of extreme prematurity. Method: The identified characteristics of 33 children aged between 4 and 5.11 months belonging to the extreme premature following policlinic of the Centro de Referencia de Salud Cordillera Oriente were compared with the characteristics of children without this background. Both groups of children reside in Santiago, Chile. The Child Apperception Test (CAT-A), a widely used projective technique in child psychological diagnosis and a specifically designed guideline aimed to the assessment of the development level of personality were used in this study. Results and Conclusions: The studied characteristics in these children were inherent and more primitive than the characteristics of children without this background. Was detected in children with a history of extreme prematurity indicators that account for the development of the structure of different children in the comparison group personality.
\end{abstract}

Key words: Extremely premature, projective techniques, personality assessment. Rev Chil Neuro-Psiquiat 2015; 53 (1): 8-17

Recibido: 29/10/2014

Aceptado: 28/01/2015

Este trabajo no contó con financiamiento externo y las autoras declaran no tener conflictos de interés.

$1 \quad$ Psicóloga y Fonoaudióloga. Departamento de Fonoaudiología. Facultad de Medicina, Universidad de Chile. Magíster en psicología clínica mención en psicodiagnóstico y evaluación forense.

2 Fonoaudióloga. Servicio de Pediatría. Centro de Referencia de Salud Cordillera Oriente.

3 Psicóloga. Magíster en psicología clínica mención en psicodiagnóstico y evaluación forense. 


\section{Introducción}

$\mathrm{A}$ nivel profesional y en el entorno familiar existe la percepción, respecto que los niños prematuros tendrían características psicológicas propias (cognitivas y afectivo-sociales) distintas a las de otros niños de la misma edad, debido a las condiciones de su nacimiento, hospitalización prolongada y posterior evolución ${ }^{1-5}$. Ellos requieren desde muy tempranamente cuidados especiales entre los que se cuentan constantes evaluaciones e intervenciones que involucran todas las dimensiones de su desarrollo ${ }^{2,3}$. Diversos estudios han dado cuenta de que estos niños presentan efectivamente dificultades cognitivas; se ha demostrado que los problemas relacionados con la memoria, las funciones ejecutivas, la atención y el lenguaje son los más frecuentes en niños que fueron prematuros extremos; es decir, niños con menos de 32 semanas de gestación y/o menos $1.500 \mathrm{~g}$ de peso $^{6-16}$. En la dimensión afectiva y social, existen investigaciones que dan cuenta de las dificultades de estos niños y de sus cuidadores, producto de la hospitalización prolongada, respecto al desarrollo de los vínculos de apego ${ }^{17-21}$.

Los eventos interpersonales positivos y negativos tempranos impactan la estructura organizacional del cerebro y la expansión de las capacidades funcionales de adaptación, existiendo una relación directa entre un entorno socio-emocional estimulante y propicio, un óptimo desarrollo cerebral y una salud mental infantil adaptativa ${ }^{22}$. En el caso de niños con antecedente de prematuridad extrema, las condiciones de nacimiento y evolución posterior constituyen experiencias adversas que podrían hacerlos más vulnerables a desarrollar estilos de personalidad más frágiles, que reflejaría las dificultades vividas en esta primera etapa de sus vidas y podrían por tanto, ser más vulnerables a presentar psicopatologías posteriores.

Investigaciones realizadas en adultos con antecedentes de haber nacido en condiciones de muy pretérmino (menos de 33 semanas de gestación) han evidenciado que durante la adolescencia y en la adultez tienen una mayor proporción de presentar hospitalización por psicosis, trastorno bipolar, depresión y otros trastornos asociados a ansiedad, que los nacidos a término ${ }^{23-25}$. Trabajos similares realizados en Estados Unidos y Noruega coinciden en el mayor riesgo de esta población de presentar enfermedades psiquiátricas ${ }^{26-28}$. Todos los sujetos estudiados tenían en común el antecedente de prematuridad con gestación menor a 33 semanas y/o muy bajo peso al nacer ${ }^{23-28}$.

Conociendo este mayor riesgo, se hace relevante investigar cuáles serían las características psicológicas de estos niños que los harían más vulnerables a presentar alteraciones psíquicas. Esto es, conocer las características de la estructura de la personalidad en desarrollo, es decir, los cimientos de su futura salud y bienestar psíquico-mental.

Para poder determinar las características distintivas y duraderas que componen la personalidad de un sujeto, es necesario referirse a este concepto. Según Kernberg, la personalidad podría definirse como "la organización molar en la cual todos los elementos se integran de manera específica dando lugar a una matriz subyacente, desde la cual se organiza y manifiesta el aparato psíquico, incorporándose los diversos procesos psíquicos, los diferentes niveles de conciencia y los aspectos del mundo interno y de la realidad externa, los aspectos ocultos así como los revelados"29.

Así, la personalidad de cada individuo estaría compuesta por una estructura que se va conformando e integrando a partir de: las experiencias tempranas, los procesos de identificación con sus figuras parentales, la forma en la que va resolviendo los conflictos propios del desarrollo y manejando los déficits que pueden haber existido.

Una persona presentaría un conjunto de rasgos que la haría situarse en un determinado punto del espectro del funcionamiento de la personalidad. Este conjunto de rasgos alude a un estilo de personalidad, lo cual permitiría entender, comparar y predecir el funcionamiento global de un determinado individuo ${ }^{30}$.

Kernberg y cols., plantean que cada estilo de personalidad se despliega en una organización estructural determinada: normal, neurótica, limítrofe alta, limítrofe baja y psicótica ${ }^{31}$. Respecto de la organización estructural en cada individuo, ésta se diferenciaría y estaría dada por tres componentes: 
la conformación de la identidad del Yo y el tipo de relación interpersonal, los mecanismos de defensa predominantemente usados y la capacidad para la prueba de realidad ${ }^{29}$.

Esta estructura se construye en un sujeto a partir de las pulsiones y las relaciones objetales en un proceso que comenzaría en una fase simbiótica con los objetos pasando por la fase de separación individuación de éstos, para llegar a la integración del sí mismo. Esto quiere decir que se han desarrollado representaciones del sí mismo y de los otros, con la posibilidad de separarse y acercarse a los objetos, sin que la relación con éstos se vea afectada irremediablemente ${ }^{32}$. Respecto de las representaciones del sí mismo, éstas pueden constituir las características más perdurables en la vida mental de un niño. Estas representaciones han sido promovidas gracias al desarrollo del afecto y las relaciones de apego, que a su vez se relacionan con el comportamiento social, la autoestima y el sentido de eficacia. Por lo anterior, la identidad, tan ligada a la adolescencia, podría pensarse también en niños pequeños ${ }^{33}$.

Partiendo de la premisa que la organización estructural de la personalidad en desarrollo es posible de ser estudiada tempranamente, se decide en esta investigación evaluar las características de cada uno de sus componentes en niños con antecedente de prematuridad extrema. Para ello, se construyó una pauta de interpretación cuantitativa denominada Pauta para la Exploración de la Estructura de Personalidad en Desarrollo (PEEPD). El propósito fue identificar las características de la organización estructural en desarrollo en estos niños, comparar estas características con las de niños sin este antecedente y detectar diferencias que pudieran hacerlos más vulnerables ya en la etapa preescolar. Se utilizaron las narraciones que se desprenden luego de la aplicación del Test de Apercepción Infantil con figuras de animales (CAT-A, prueba proyectiva psicoanalítica para niños, de interpretación cualitativa $)^{34}$.

\section{Material y Método}

\section{Diseño muestral}

La presente investigación se planteó como un estudio descriptivo-comparativo, transversal, no experimental y con un enfoque cuantitativo. La muestra estuvo compuesta por 66 niños de entre 4 y 5 años 11 meses, escolarizados al momento de la evaluación. Se dividieron en dos categorías: a) niños con antecedente de prematuridad extrema (17 niños y 16 niñas de menos de 32 semanas de gestación y/o menos de $1.500 \mathrm{~g}$ de peso al momento de nacer), asistentes al policlínico de seguimiento del prematuro extremo del Centro de Referencia en Salud (CRS Cordillera Oriente) y b) niños sin antecedente de prematuridad extrema (15 niños y 18 niñas), ni hospitalizaciones tempranas ni prolongadas, pertenecientes a la misma comuna y nivel socioeconómico que el de los niños del grupo en estudio. Se excluyeron de esta investigación a niños con patología neurológica severa, trastorno severo del lenguaje y factores psicosociales extremos. Ambos grupos de niños pertenecían a la ciudad de Santiago de la Región Metropolitana de Chile.

Antes de seleccionar al grupo en estudio, se contó con la autorización del comité de ética del CRS Cordillera Oriente, quienes entregaron recomendaciones que se incorporaron al presente trabajo y dieron el permiso correspondiente para iniciar esta investigación.

\section{Instrumento confeccionado para esta investigación}

Con el fin de transformar los resultados cualitativos obtenidos de la aplicación del CAT-A, en datos cuantitativos, se confeccionó una pauta basada en el marco teórico de los autores originales del test $^{34}$, al cual se integraron: el modelo de la organización estructural de la personalidad del Dr. Otto Kernberg ${ }^{33}$ y los aportes en cuanto al desarrollo de la personalidad en niños y adolescentes de la Dra. Paulina Kernberg y sus colaboradores ${ }^{31}$.

Esta pauta se denominó Pauta para la Exploración de la Estructura de Personalidad en Desarrollo (PEEPD) (Tabla 1) y se construyó empleando como base las categorías propuestas por Bellak y Bellak $^{34}$ para la interpretación de cada una de las láminas del CAT-A. Se determinaron múltiples indicadores específicos para cada categoría y se le asignó a cada indicador los valores resultantes 
Tabla 1. Distribución de indicadores en categorías y ejes que componen la Pauta para la Exploración de la Estructura de Personalidad en Desarrollo (PEEDP)

\begin{tabular}{|c|c|c|c|}
\hline Eje & Categoría & Indicador & Sub indicador \\
\hline I. Temática & $\begin{array}{l}\text { Correspondencia } \\
\text { Desenlace }\end{array}$ & $\begin{array}{l}3 \\
3\end{array}$ & \\
\hline II. Identidad y R. interpersonales & $\begin{array}{l}\text { Yo } \\
\text { Súper Yo } \\
\text { R. Interpersonales }\end{array}$ & $\begin{array}{l}5 \\
2 \\
4\end{array}$ & $\begin{array}{l}11 \\
20\end{array}$ \\
\hline III. Mecanismos de defensa & $\begin{array}{l}\text { Impulsos y Ansiedad } \\
\text { Normales } \\
\text { Neuróticos } \\
\text { Limítrofes } \\
\text { Psicóticos }\end{array}$ & $\begin{array}{r}4 \\
9 \\
13 \\
7 \\
10\end{array}$ & 10 \\
\hline IV. Prueba de realidad & $\begin{array}{l}\text { Diferenciación } \\
\text { Pensamiento } \\
\text { Percepción } \\
\text { Interpretación }\end{array}$ & $\begin{array}{l}1 \\
2 \\
2 \\
1\end{array}$ & \\
\hline
\end{tabular}

posibles. Se obtuvo así una amplia matriz de indicadores (nominales dicotómicos, categoriales y ordinales) con sus posibles valores cualitativos y se asignó un valor numérico a cada uno de ellos.

A modo de ejemplo, para cada lámina los autores proponen evaluar el desarrollo del yo a través de la observación del héroe. Para esta categoría se establecieron 5 indicadores: representación del yo, fortaleza del yo, control de impulsos, capacidad de empatizar y visión de sí mismo. Para el indicador fortaleza del yo se establecen 3 posibles valores: débil (1), moderado (2) y fuerte (3). Finalmente, las características para evaluar el desarrollo del yo se integran en el componente identidad y relaciones interpersonales propuesto por Kernberg ${ }^{33}$.

Estos indicadores se seleccionaron y organizaron en función de los componentes de la estructura de personalidad propuesta por Otto Kernberg considerando los aportes de Paulina Kernberg en relación al desarrollo de las diferentes estructuras de personalidad durante la niñez y la adolescencia ${ }^{31}$.

Con el fin de aportar a la validez de contenido del instrumento se sometió la PEEPD a un pilotaje, aplicándola a cuatro protocolos de niños preescolares con edades entre 4 y 5 años 11 meses con desarrollo típico o normal. A través de este procedimiento fue posible definir criterios conjun- tos para la aplicación de la pauta y completar las indicaciones para la cuantificación de cada uno de los indicadores y subindicadores. En este pilotaje se contó además con la ayuda de una especialista en psicodiagnóstico infanto-juvenil y dos juezas expertas ajenas a esta investigación, quienes contribuyeron en la delimitación e interpretación de los indicadores de la pauta.

Finalmente, esta pauta quedó constituida en 94 indicadores organizados en torno a 15 categorías y 4 ejes principales.

\section{Procedimientos}

Luego de la selección definitiva de los grupos se procedió a aplicar el CAT-A y transcribir cada una de las narraciones. Se realizó al análisis cualitativo de las narrativas y se traspasaron estas interpretaciones a la Pauta para la Exploración de la Estructura de Personalidad (PEEPD) como datos cuantitativos. A continuación, se procedió a determinar la proporción en que se presentaba cada indicador por lámina y por sujeto, obteniéndose el estadístico descriptivo "media" para cada uno de los grupos (en estudio y comparativo). Finalmente, se exportó la base de datos desde la planilla Excel, al programa estadístico SPSS (Statiscal Package for Social Sciences) versión 21.0 
Tabla 2. Principales características del grupo en estudio, ordenados según componentes de la estructura de personalidad

\begin{tabular}{|ll|}
\hline Componente & Niños con antecedente de prematuridad extrema \\
Identidad y relaciones interpersonales & Yo débil \\
& $\begin{array}{l}\text { Imagen sí mismo inseguro o temeroso } \\
\text { Descontrol de impulsos } \\
\text { Súper yo agresivo y severo }\end{array}$ \\
Mecanismos de defensa & $\begin{array}{l}\text { Alta ansiedad de daño y muerte } \\
\text { Mecanismos de defensa predominantemente de tipo limítrofes }\end{array}$ \\
Prueba de realidad & Tendencia a la pérdida del sentido de la realidad \\
\hline
\end{tabular}

Nota: Todos estos indicadores presentaron diferencias significativas respecto del grupo comparativo. $\mathrm{p}<0,05$.

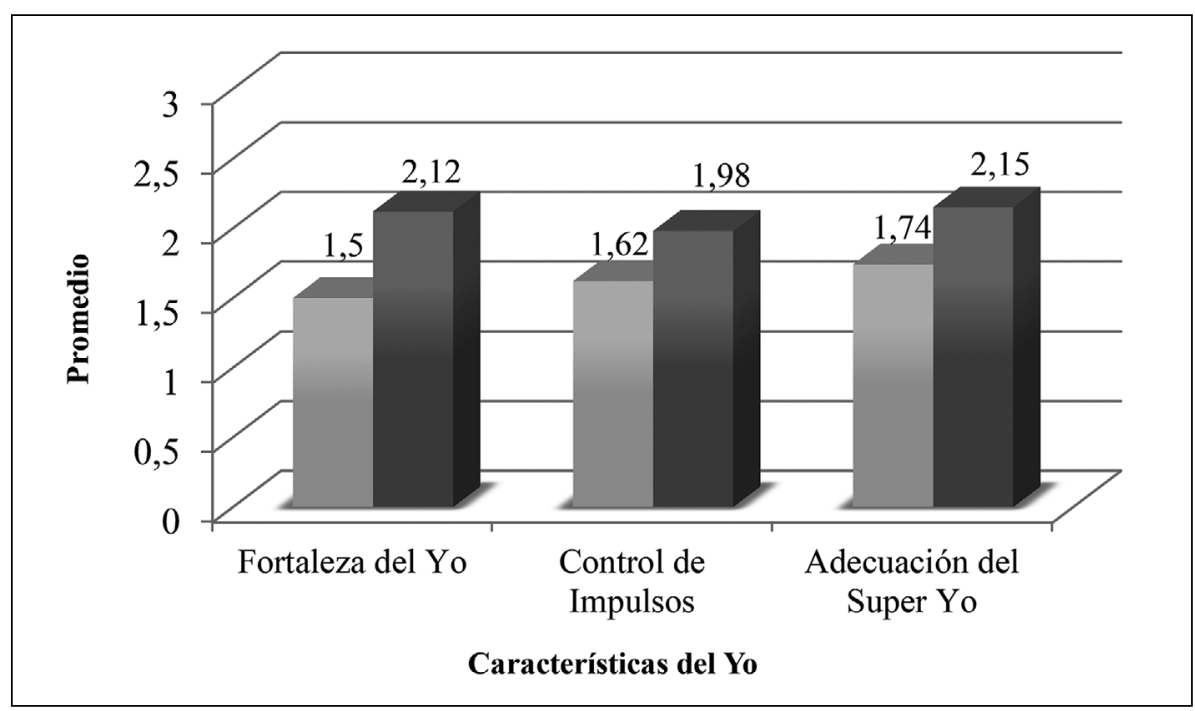

Figura 1. Características del Yo en el grupo en estudio y el grupo comparativo. Nota: Los indicadores se evalúan como variables continuas que toman valores entre 1 y 3 puntos. para Windows, aplicándose la prueba estadística paramétrica T. Se consideró como nivel de significancia $\mathrm{p}<0,05$.

\section{Resultados}

A continuación se presentan los resultados ordenados de acuerdo a los componentes de la estructura de personalidad que permitieron la caracterización de los grupos (Tabla 2):

Desde el componente identidad y relaciones interpersonales, se puede afirmar que los niños del grupo de prematuros extremos al igual que el grupo comparativo, identifican en la mayoría de sus narraciones al personaje principal o héroe, incluyendo en ellas a figuras parentales, pares y otros personajes agregados en igual medida. Todos los niños manifiestan una buena capacidad de empatizar con los personajes. A diferencia del grupo comparativo, los niños del grupo en estudio presentan una imagen de sí mismos en que destacan características como inseguridad o temor y el sentirse agredidos y abandonados. Sus relatos manifiestan una tendencia hacia el descontrol de impulsos, así como hacia la debilidad yoica. El Súper Yo en su mayoría se aprecia como débil, agresivo y severo (Figura 1).

Respecto de las figuras parentales, los niños con antecedente de prematuridad extrema las 


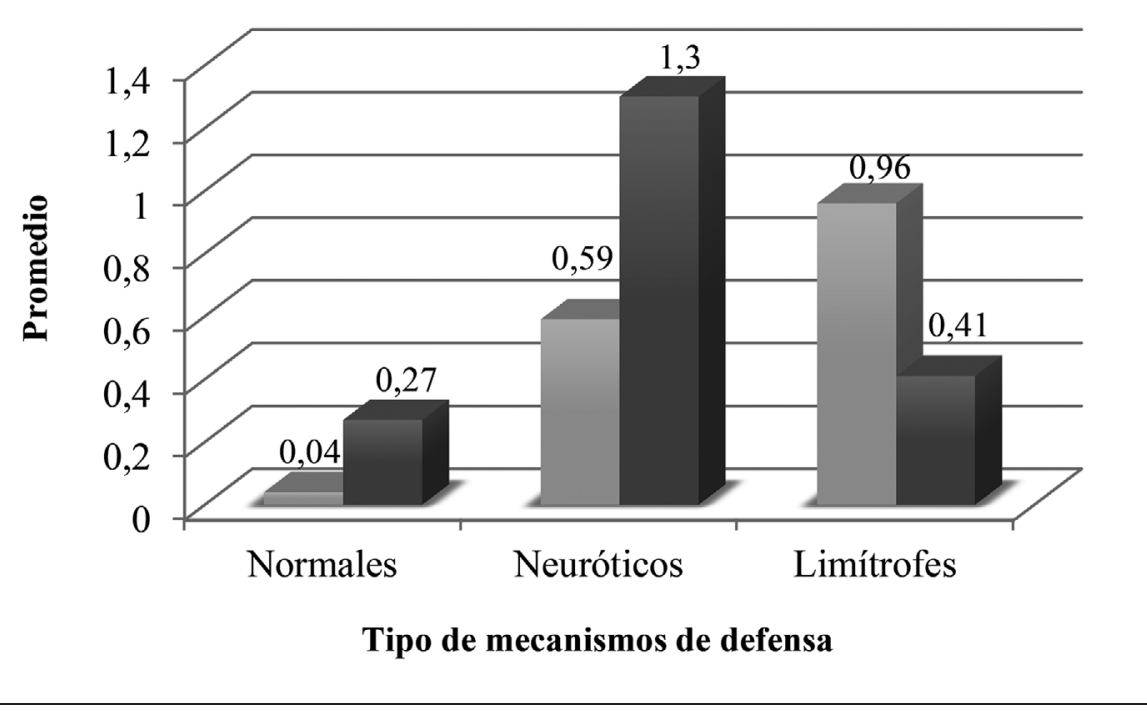

Figura 2. Comparación de los mecanismos de defensa empleados por el grupo en estudio y grupo comparativo. Nota: Todas las diferencias resultaron significativas. $\mathrm{p}<0,05$. perciben como ineficientes; con sus pares, cuando aparecen, tienden a relacionarse más en la complicidad que en la rivalidad. Respecto de la visión del entorno, lo perciben mayoritariamente como amenazante, terrorífico e impredecible.

Desde el segundo componente de la estructura de personalidad, los mecanismos de defensa, se puede afirmar que en el grupo en estudio a diferencia del grupo comparativo, los impulsos son intensos y predominantemente agresivos. La ansiedad surge principalmente ante situaciones de posible daño, de muerte y de ser devorado.

Los mecanismos de defensa mayormente utilizados por el grupo en estudio, son los mecanismos de tipo limítrofe: negación limítrofe y devaluación primitiva. Los mecanismos de tipo neurótico, están significativamente menos presentes en este grupo. Los mecanismos normales tienen un porcentaje de aparición muy bajo. No hay presencia de mecanismos de defensa de tipo psicóticos en ninguno de los grupos (Figura 2).

Desde el componente prueba de realidad, destaca que los niños del grupo en estudio presentan una menor capacidad de diferenciación del sí mismo respecto al entorno. Respecto a su capacidad para interpretar los eventos externos, se observa una tendencia a la pérdida del sentido de la rea- lidad con un mayor grado de subjetividad en sus relatos.

Las características que aparecen luego del análisis de los componentes de la estructura de personalidad en desarrollo del grupo de niños con antecedente de prematuridad extrema son coincidentes con lo descrito por P. Kernberg respecto a las características de niños con estructura de personalidad limítrofe. Ella define estas características destacando la angustia de aniquilación, niveles inestables de funcionamiento yoico, mecanismos de defensa primitivos, funcionamiento superyoico ineficaz, alteraciones en la identidad, actividad fantasiosa significativa, incapacidad para expresar moduladamente sus sentimientos y un pobre control de impulsos, con estados no predecibles ${ }^{31}$.

\section{Discusión}

Los hallazgos de esta investigación respecto a que en el desarrollo de la estructura de personalidad de los niños con antecedente de prematuridad extrema existen características propias y más primitivas que los niños sin este antecedente, son muestra evidente de un desarrollo psíquico no típico y diferente en este grupo de niños. 
Lo anterior tiene un respaldo con lo descrito en la literatura científica respecto a que la vulnerabilidad biológica de los niños prematuros podría perturbar su desarrollo psíquico y relacional ${ }^{21}$. Debido a estas condiciones al nacer, pueden producirse estados traumáticos en la infancia que desencadenan alteraciones psicobiológicas, las cuales impactan la cognición, el afecto y el comportamiento ${ }^{22}$.

Las narraciones de los niños del grupo en estudio fueron particularmente diferentes y llamativas ya desde la observación clínica, respecto del grupo comparativo. En la relación establecida en la instancia evaluativa, la vivencia transferencial estuvo cargada de intensas emociones, pues el contenido de los relatos resultó impactante y sorpresivo, con marcada violencia y angustia, sin concordancia afectiva, es decir, con una clara desafectación.

Lo anterior se confirma con los resultados del análisis estadístico realizado en esta investigación, en donde resultó significativa la aparición de relatos marcados por un entorno amenazante y muchas veces terrorífico, con desenlaces desdichados sin aparente sentido. Las ansiedades que prevalecen en estos niños son ante un posible daño, lesión y/o muerte y para enfrentarlas requieren emplear mecanismos de defensa masivos e intensos, con altos niveles de omnipotencia, que resultan eficaces, pero insuficientes para el manejo de estas ansiedades.

Si se consideran nuevamente las condiciones de nacimiento y posterior evolución, se puede comprender que estas potentes ansiedades podrían haber estado ya presentes en sus padres al momento de nacer, asociados también con intensos sentimientos de culpabilidad y de ambivalencia ligados al embarazo, que podrían aumentar debido al paso por tratamientos largos y difíciles de reproducción asistida, o bien debido a un embarazo no planificado, lo que ocurre con frecuencia en las familias de estos niños. Ante esto, los niños necesitan protegerse y muchas veces lo que se observa es la hiperactividad, que aparece en primer plano, a modo de defensa maníaca que enmascara el sufrimiento depresivo ${ }^{21}$.

Las características encontradas en estos niños son similares a las mencionadas por otros autores que han estudiado desarrollo de estructura de personalidad en niños con diversas alteraciones psíquicas. La impulsividad es uno de los rasgos más distintivos y se manifiesta de manera clara en la alta intensidad y dificultad en el control de los impulsos ${ }^{31}$, así como también la falta de tolerancia a la frustración, siendo lo central, la dificultad en la regulación de los afectos ${ }^{35}$.

Todo lo anteriormente descrito da cuenta de la necesidad de intervenir tempranamente respecto de los aspectos afectivos, relacionales y sociales en estos niños y niñas, para favorecer el desarrollo de diferentes estilos de enfrentamiento a los problemas, propiciar una percepción más realista del contexto y así favorecer el proceso de simbiosis, diferenciación y posteriormente de integración del sí mismo.

Finalmente, se hace también muy necesario realizar futuras investigaciones en torno al desarrollo de la personalidad en niños con antecedente de prematuridad, dado el aumento progresivo en la sobrevida y porque tienen mayor riesgo de presentar alteraciones de salud mental en la adolescencia y adultez. De allí la importancia de la intervención cuanto antes en el tiempo, tanto a ellos como a sus familias. 


\begin{abstract}
Resumen
Introducción: Los niños prematuros extremos son una población de alta vulnerabilidad biológica producto de las condiciones de su nacimiento: hospitalización temprana y prolongada con riesgo vital. Estos niños presentan con frecuencia dificultades cognitivas, pero también afectivo -sociales y en el establecimiento de los vínculos de apego. Por tanto, se podría desprender que habría consecuencias desfavorables en el desarrollo de su estructura de personalidad. Dado lo anterior, el objetivo de este estudio fue identificar las características propias de los componentes de la estructura de la personalidad en desarrollo de niños con antecedente de prematuridad extrema. Método: Se compararon las características identificadas en 33 niños de entre 4 y 5 años 11 meses, pertenecientes al policlínico de seguimiento de prematuros extremos del Centro de Referencia de Salud Cordillera Oriente, con las de niños sin este antecedente. Ambos grupos de niños pertenecían a la ciudad de Santiago de Chile. Se utilizó el Test de Apercepción Infantil (CAT-A), técnica proyectiva de amplio uso en el psicodiagnóstico infantil y además una pauta confeccionada especialmente para esta investigación que tenía como propósito la evaluación de la personalidad en desarrollo. Resultados y Conclusiones: Las características de los componentes de la estructura de personalidad en estos niños que fueron estudiados son propias y más primitivas que las de niños sin este antecedente. Se detectó en los niños con antecedente de prematuridad extrema indicadores que dan cuenta de un desarrollo de la estructura de personalidad diferente al de los niños del grupo comparativo.
\end{abstract}

Palabras clave: Prematuro extremo, técnicas proyectivas, evaluación de personalidad.

\section{Referencias bibliográficas}

1. Marlow N. Outcome following extremely preterm birth. Current Obstetrics \& Gynaecology 2006; 16 (3): 141-6.

2. Morgues M. (2009). Seguimiento del prematuro en Chile. Revisado el 14/03/2014 desde internet: http://www.neovidas.cl/ppt/seguimiento-prematuros-chile.pdf

3. Hospital Santiago Oriente Dr. Luis Tisné Brouse (2010). Guías de práctica clínica Revisado el 22/02/2014 desde internet: http://www.horiente. cl/29_guias_clinicas.html

4. Sastre-Riba S. Prematuridad: Análisis y seguimiento de las funciones ejecutivas. Rev Neurol 2009; 48 (2): 113-8.

5. Narberhaus A, Segarra D. Trastornos neuropsicológicos y del neurodesarrollo en el prematuro. Anales de psicología 2004; 20 (2): 317-26.

6. Anderson P, Doyle L. Cognitive and educational deficits in children born extremely preterm. Semin Perinatol 2008; 32: 51-8.

7. Rickards A, Kitchen W, Doyle L, Ford G, Kelly E, et al. Cognition, school performance, and behaviour in very low birth weight and normal birth weight children at 8 years of age: a longitudinal study. J Dev Behav Pediatr 1993; 14 (6): 363-8.

8. Casasbuenas OL. Seguimiento neurológico del recién nacido pretérmino. Rev Neurol 2005; 40 (1): 65-7.

9. Narberhaus A, Segarra MD, Pueyo R, Botet, F, Junqué $\mathrm{C}$. Disfunciones cognitivas a largo plazo en sujetos prematuros con hemorragia intraventricular. Rev Neurol 2008; 47 (2): 57-60.

10. Anderson P, Doyle L. Executive functioning in school-aged children who were born very preterm or with extremely low weight birth in the 1990 . Pediatrics 2004; 114 (1): 50-7.

11. Ishii C, Shizue C, Isotani S, Perissinoto J. Caracterização de comportamentos lingüisticos de 
crianças nascidas prematuras aos quatro anos de idade. Rev CEFAC 2006; 8 (2): 147-54.

12. Peña M, Pittaluga E, Farkas Ch. Adquisición fonológica en prematuros. Rev Neurol 2010; 50 (1): 12-8.

13. Rizzotto C, Wetters M, Lahorgue M. Clinical assessment of language development in children at age 3 years that were born pretem. Arq Neuropsiquiatric 2006; 64 (4): 926-31.

14. Sansavini A, Guarini A, Alessandroni R, Faldella G, Giovanelli G, Salvioli G. Are early grammatical and phonological working memory abilities affected by preterm birth? J Commun Disord 2007; 40: 239-56.

15. Maggiolo M, Varela V, Arancibia C, Ruiz F. Dificultades del lenguaje en niños preescolares con antecedente de prematuridad extrema. Rev Chil Pediatr 2014; 85 (3): 319-27.

16. Taylor G, Klein N, Minich N, Hack M. Verbal memory deficits in children with less than 750 g birth weight. Child Neuropsychol 2000; 6 (1): 49-63.

17. Spittle A, Treyvaund K, Doyle L, Roberts G, Lee $\mathrm{K}$, Inder $\mathrm{T}$, et al. Early Emergence of Behavior and Social-Emotional. Problems in Very Preterm Infants. J Am Acad Child Adolesc Psychiatry 2009; 48 (9): 909-18.

18. Loe I, Lee E, Luna B, Feldman H. Behavior problems of 9-16 year old preterm children: Biological, sociodemographic, and intellectual contributions. Early Human Development 2011; 87: 247-52.

19. Muñoz P, Sánchez M del C, Méndez I, Mandujano M. Sistemas diádicos y secuelas al año de edad en niños con daño neurológico perinatal. Perinatol Reprod Hum 2003; 17: 10-9.

20. Rossel C, Carreño T, Maldonado M. Afectividad en madres de niños prematuros hospitalizados. Un mundo desconocido. Rev Chil Pediatr 2002; 73, 1 : 15-21.

21. González F. Nacer de nuevo: La crianza de los niños prematuros. La relación temprana y el apego. Cuadernos de psiquiatría y psicoterapia del niño y del adolescente 2009; 48: 61-80.

22. Schore A. The effects of early relational trauma on right brain development, affect regulation, and infant mental health. Infant Mental Health Journal 2001b; 22 (1-2): 201-69.

23. Walshe M, RifkinL, Rooney M, Healy E, Nosarti $\mathrm{Ch}$, Wyatt J, et al. Psychiatric disorder in young adults born very preterm: Role of family history. European Psychiatry 2008; 23: 527-31.

24. Nosarti Ch. Preterm birth and psychiatric outcome in adolescence and early adulthood: a study using a Swedish National Registred. Schizophrenia Research 2008; 102 (1-3, Supplement 2): 1-279.

25. Nosarti Ch, Reichenberg A, Murray R, Cnattingius S, Lambe M, Yin L, et al. Preterm Birth and Psychiatric Disorders in Young Adult Life. Arch Gen Psychiatry Published 2012; 10: 407-28.

26. Hack M, Flannery, D, Schluchter M, Cartar L, Borawski E, Klein N. Outcomes in young adulthood for very low birth weight infants. The New England Journal of Medicine 2002; 346 (3): 14957.

27. Lund L, Vik T, Skranes J, Lydersen S, Brubakk AM, Indredavik M. Low birth weight and psychiatric morbidity; stability and change between adolescence and young adulthood. Early Human Development 2012; 88: 623-9.

28. Elgen IB, Holsten F, Odberg MD. Psychiatric disorders in low birthweight young adults. Prevalence and association with assessments at 11 years Article in press. Available on lineat Sci Verse Science Direc 2012. Revisado el 02/06/2014 desde internet http://dx.doi.org/10.1016/j.eurpsy.

29. Kernberg O. Trastornos graves de la personalidad. Estrategias psicoterapéuticas. México: Editorial El Manual Moderno. 1987.

30. Rapaport E. Psicodiagnóstico, Juicio Clínico y Cambio Psíquico. En Riquelme R, Thumala E. Avances en Psicoterapia y Cambio Psíquico. Santiago: Sociedad Chilena de Salud Mental. 2005.

31. Kernberg P, Weiner A, Bardenstein K. Trastornos de personalidad en niños y adolescentes. México: Editorial El Manual Moderno. 2002.

32. Kernberg P. Capítulo 16: Psicología del desarrollo según Margaret Mahler y su importancia en los trastornos de personalidad. En: Riquelme R \& Oksenberg A. (Eds). Trastornos de personalidad: hacia una mirada integral (pp. 283-92) Chile: Salesianos. 2003. 
33. Kernberg O. Capítulo 11: Una teoría psicoanalítica de los trastornos de personalidad. En: Riquelme R \& Oksenberg A. (Eds). Trastornos de personalidad: hacia una mirada integral (pp. 205-32) Chile: Salesianos. 2003.

34. Bellak L, Bellak S. Test de Apercepción Infantil con figuras de animales: CAT-A. (2 ${ }^{\text {a }}$ Ed.) Buenos Aires, Argentina: Editorial Paidós. 2011.
35. Quiroga F. Estudio de la Organización de la Estructura de Personalidad en Niños entre 5 y 8 años de edad, que presentan un Trastorno de Inestabilidad Emocional de tipo Limítrofe, a través del Test de Rorschach. Tesis para optar al grado de magíster en psicología mención psicología clínica infanto-juvenil. Universidad de Chile. Santiago, Chile. 2005.

Correspondencia:

Virginia Varela-Moraga

Facultad de Medicina. Universidad de Chile.

Av. Independencia 1027

Independencia, Santiago, Chile.

Fono fijo: 562-29786604

E-mail: vvarela@med.uchile.cl 